\title{
ARTICLE Neurocognitive effects of umami: association with eating behavior and food choice
}

\author{
Greta Magerowski ${ }^{1}$, Gabrielle Giacona ${ }^{1}$, Laura Patriarca ${ }^{1}$, Konstantinos Papadopoulos ${ }^{1}$, Paola Garza-Naveda ${ }^{2}$, \\ Joanna Radziejowska ${ }^{2}$ and Miguel Alonso-Alonso (iD ${ }^{1}$
}

Free glutamate, a key substance underlying the umami taste of foods, fulfills a number of physiological functions related to energy balance. Previous experimental studies have shown that intake of a broth or soup supplemented with monosodium glutamate (MSG) prior to a meal can decrease appetite and food intake, particularly in women with propensity to overeat and gain weight. In this study, we examined potential neurocognitive mechanisms underlying this effect. We evaluated changes after intake of a chicken broth with or without MSG added (MSG+/MSG-) in a sample of healthy young women. Subjects were assessed with a food-modified computerized inhibitory control task, a buffet meal test with eye-tracking, and brain responses during a food choice paradigm evaluated with functional neuroimaging. We found evidence for improvement in key parameters related to inhibitory control following intake of the MSG + broth, particularly in subjects with high levels of eating disinhibition, who also showed lower intake of saturated fat during the meal. Additionally, consumption of the MSG+ broth led to a reduction of the rate of fixation switches between plates at the meal, and increased engagement of a brain region in the left dorsolateral prefrontal cortex previously associated with successful self-control during dietary decisions. Altogether, these results, while preliminary, suggest potential facilitating effects of glutamate (MSG) on cognitive executive processes that are relevant for the support of healthy eating behaviors and food choice.

Neuropsychopharmacology (2018) 43:2009-2016; https://doi.org/10.1038/s41386-018-0044-6

\section{INTRODUCTION}

Umami, a Japanese word to express a delicious, savory meal, represents one of the five basic tastes, together with sweet, salty, bitter, and sour. A key determinant of umami taste is glutamate, a naturally occurring non-essential amino acid that can be found in nearly all foods, including many vegetables and especially foods high in protein such as dairy products, fish and meat. Foods often used for their flavoring properties in traditional cuisines around the world, such as mushrooms, tomatoes, Parmesan cheese or fermented products, have high levels of free glutamate. Monosodium L-glutamate (MSG) is a sodium salt of glutamate that became commercially available after the isolation, characterization and subsequent synthesis of the umami component in the early 1900 's [1, 2]. MSG is widely used as a food additive to reduce cooking and meal preparation time and to enhance the sensory attributes of food.

Beyond its role as an additive, detailed physiological studies in animals suggest that glutamate is also involved in processes that are relevant for energy homeostasis, such as digestion, dietinduced thermogenesis, and the development of flavor preferences [3-5]; behavioral and neurocognitive effects have been less studied. Glutamate is sensed in the oral cavity and gastrointestinal tract mostly via T1R1 + T1R3 and metabotropic glutamate receptors (mGluR) [6]. Information about its presence is conveyed from the stomach to the brain through a specific vagus-nervemediated signaling pathway that may serve as a key indicator of protein content in ingested food [5, 7-9]. Studies with functional magnetic resonance imaging (fMRI) in rodents have shown that following intragastric infusion of glutamate (MSG) an array of brain regions such as thalamic nuclei, insular cortex, limbic system, and the hypothalamus become activated but, unlike following the ingestion of glucose, no changes occur in the nucleus accumbens [10]. While this is compatible with the absence of weight gain in rats when they are given free access to a solution with MSG [11], the translation of these findings to and implications for humans remain largely unexplored.

A number of studies have examined short-term effects of MSG intake administered in the form of a soup or broth preload in human subjects [12-19]. Adding MSG increases the palatability of food and has the potential to increase consumption; however, the evidence for both short-term and long-term changes in appetite and food intake related to MSG is mixed [20]. Savory preloads that contain MSG can decrease hunger and desire to snack in women [19] and can decrease preference for high-fat and sweet foods in a subsequent meal [13]. The reduction of appetite and food intake is stronger with savory preloads than with sweet or bland preloads, and is more prominent in women with high eating disinhibition or with excess weight $[12,17]$. fMRI studies in humans have shown that taste responses to MSG, compared with glucose, include more activation in the dorsal sector of the anterior cingulate cortex [21], an area that plays a key role in complex cognitive processes related to behavioral adjustment [22]. A full

\footnotetext{
'Laboratory of Bariatric and Nutritional Neuroscience, Center for the Study of Nutrition Medicine, Department of Surgery, Beth Israel Deaconess Medical Center, Harvard Medical School, Boston, MA, USA and ${ }^{2}$ Harvard-Thorndike Clinical Research Center, Beth Israel Deaconess Medical Center, Harvard Medical School, Boston, MA, USA

Correspondence: Miguel Alonso-Alonso (malonso@bidmc.harvard.edu)
}

Received: 24 November 2017 Revised: 25 February 2018 Accepted: 1 March 2018

Published online: 30 March 2018 


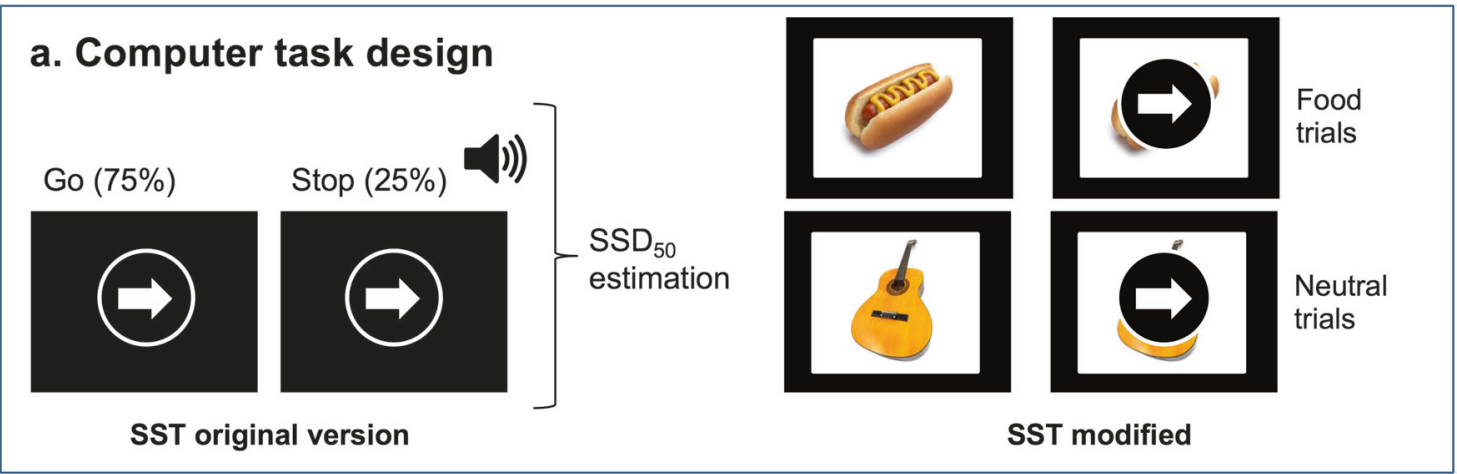

\section{b. Buffet meal test}
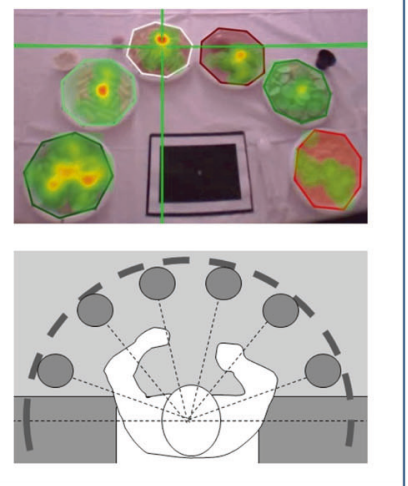

c. fMRI task

Run 1: HEALTH rating

Run 2: TASTE rating

Run 3: $\mathrm{CHOICE} \longrightarrow$
Self-regulation trials

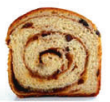

e.g. Neutral Food

(Health:3 Taste:3) vs

Seeds (Health:5 Taste:2)

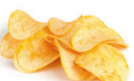

e.g. Neutral Food

(Health:3 Taste:3) vs

Chips (Health:1 Taste:5)

Non-self-regulation trials

\section{d. Broth}

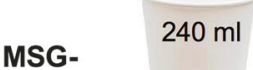

$3 g$ protein

$1 \mathrm{~g}$ sugar

$750 \mathrm{mg}$ sodium

MSG+

$3 \mathrm{~g}$ protein

$1 \mathrm{~g}$ sugar

$1.44 \mathrm{~g} \mathrm{MSG}$

$750 \mathrm{mg}$ sodium

Fig. 1 Study procedures. Illustration of all the components involved in the protocol: a Stop-signal task, original version and modified version with images of food and neutral items in the background. b Buffet meal test with eye tracking. The top image shows a general view of the buffet meal test with an overlaid heat map. The bottom image represents a diagram of the buffet meal setup, subject position and distribution of plates around the table. c Overview of the fMRI food choice task: health, taste and choice runs and illustration of two trials involving selfregulation. d Broth composition for the two conditions. For details see supplementary materials and methods section

understanding of these effects and underlying mechanisms is warranted.

In the present study, we examined the possibility that intake of MSG may influence human eating behavior through neurocognitive effects. Based on the above findings, it is plausible that glutamate sensing in the stomach by the vagus nerve may lead to upstream activation of brainstem noradrenergic projections to the cortex [23], with the potential of bottom-up modulation of cognitive-executive processes as complex as decision-making [2426]. To examine this possibility, we designed a randomized, double blind, crossover experiment where we evaluated neurocognitive and eating behavior effects following intake of a broth supplemented with MSG in a group of healthy women. Effects along the brain-cognition-behavior spectrum were measured using an inhibitory control task modified for food, a buffet meal test where subjects wore a portable eye-tracking system and an fMRI paradigm to examine brain activation during dietary decisions. We hypothesized that, via taste receptor signaling and vagus nerve afferents, MSG could influence neurocognitive pathways in a bottom-up manner leading to an enhancement in the activity of brain regions and cognitive processes related to executive function, specifically aspects of inhibitory control. We also predicted that the intake of MSG would lead to increased engagement of brain regions previously associated with selfregulation during food choice, specifically the dorsolateral prefrontal cortex (DLPFC) [27]. We also hypothesized that, based on past findings in the human eating behavior literature, the anticipated effects of MSG on neurocognition would be prominently influenced by individual characteristics related to propensity to overeat and gain weight (eating disinhibition).

\section{MATERIALS AND METHODS}

\section{Subjects}

A total of 41 healthy, non-obese women were recruited through an internet advertisement, available to current students at a local university. Interested participants were invited to the laboratory, where they were screened for inclusion in the study. Participants were between 18 and 30 years old, with a self-reported normal $\mathrm{BMI}$, no food allergies, intolerances or restrictions, no history of smoking, no MRI contraindications, either no myopia or myopia for which prescribed glasses or contact lenses measured less than -1.5 diopters, and no current pregnancy. A total of 35 participants (mean age: $20.5 \pm 0.3$ years, mean BMl $22.1 \pm 0.4 \mathrm{~kg} / \mathrm{m}^{2}$ ) were finally enrolled and randomized to the two study groups, consisting of buffet meal or fMRI (see below). The study included only women, as its intention was to build upon the results of previous investigations with MSG and also to reduce well-known outcome variability in this area of research related to gender [19]. From the original 35 subjects, 5 subjects were excluded from the analysis due to incomplete visits or inconsistent fasting hours, leaving a final number of 30 participants for the computer tasks, 15 for the buffet meal tests, and 14 for the fMRI sessions.

\section{Study design}

Subjects were required to come to the laboratory on four different days, aside from the baseline visit, over a period of 10 days. During the initial 2 days (common to all participants), they performed a classical and a food-modified version of the stop-signal task (SST) for the assessment of inhibitory control under two different conditions: (a) following intake of a control broth (MSG-) and (b) following intake of a broth supplemented with MSG (MSG+). In 
Table 1. Summary of findings in the stop-signal task

\begin{tabular}{|c|c|c|c|c|c|c|c|c|}
\hline & \multicolumn{4}{|l|}{ MSG- } & \multicolumn{4}{|c|}{ MSG +} \\
\hline & \multicolumn{2}{|c|}{ Pre-broth } & \multicolumn{2}{|c|}{ Post-broth } & \multicolumn{2}{|c|}{ Pre-broth } & \multicolumn{2}{|c|}{ Post-broth } \\
\hline & Mean & SD & Mean & SD & Mean & SD & Mean & SD \\
\hline \multicolumn{9}{|l|}{ SSRT (ms) } \\
\hline Food & 157.5 & 38.3 & 164.3 & 41.7 & 153.9 & 29.2 & 155.1 & 32.6 \\
\hline Neutral & 157.2 & 34.3 & 160.4 & 40.0 & 156.4 & 27.9 & 153.6 & 31.0 \\
\hline \multicolumn{9}{|c|}{ Correct stops (\%) } \\
\hline Food & 53.9 & 12.2 & 57.4 & 11.6 & 54.0 & 12.2 & 56.3 & 12.1 \\
\hline Neutral & 50.6 & 12.1 & 56.0 & 13.6 & 52.3 & 11.2 & 56.2 & 12.8 \\
\hline \multicolumn{9}{|c|}{ Go RT (ms) } \\
\hline Food & 473.5 & 71.1 & 480.3 & 79.8 & 471.5 & 59.7 & 472.8 & 61.4 \\
\hline Neutral & 473.2 & 69.9 & 476.4 & 79.4 & 474.0 & 56.1 & 471.3 & 60.0 \\
\hline \multicolumn{9}{|l|}{ ICV } \\
\hline Food & 0.20 & 0.06 & 0.19 & 0.06 & 0.20 & 0.06 & 0.19 & 0.06 \\
\hline Neutral & 0.21 & 0.06 & 0.19 & 0.06 & 0.21 & 0.05 & 0.18 & 0.05 \\
\hline
\end{tabular}

brief, both versions of the SST consisted of a 75:25\% mix of go and stop trials. Subjects were to respond as quickly as possible (go trials) to the display of a left- or right-pointing arrow on the computer screen by pressing the corresponding arrow key on the keyboard. They were to withhold this response (stop trials) if, briefly following the appearance of the arrow, a tone sounded. The delay of the tone after the display of the arrow was automatically adjusted during the task such that subjects would correctly inhibit responses $50 \%$ of the time. On each of the two computer task days, subjects started by performing a classical version of the SST. Based on that performance we obtained a task parameter- the average stop-signal delay-at which the subject had a $50 \%$ probability of stop accuracy performance $\left(\mathrm{SSD}_{50}\right)$. This value was used in the two subsequent food-modified SSTs, which subjects preformed pre- and post-broth. In the food-modified SST, images of food or neutral items preceded each arrow and remained onscreen until the end of the trial. The order of food and neutral images was randomized. We evaluated the following parameters in the food-modified SST: psychomotor performance (mean go reaction times), response inhibition [stop signal reaction time (SSRT), reflecting the time that it takes for a subject to inhibit a response that has been initiated (main outcome measure); percentage of successful stops], and response time variability (intra-individual coefficient of variation (ICV), a measure of attentional fluctuation during performance). For further detail about the task and data analysis, see Supplementary Materials and Methods section.

Subsequently, subjects were randomized (1:1 allocation ratio) to undergo either two sessions of a multi-choice buffet meal test with eye-tracking or two sessions of an fMRI food choice paradigm based on previous research [27]. Subjects were evaluated after intake of the broth (MSG-/MSG+ conditions) during these two additional visits as well. Full details of the buffet meal test, fMRI paradigm and data analysis are included in Supplementary Materials and Methods section. Figure 1 illustrates each of the components involved in the study protocol. The order of broth conditions was randomized and counterbalanced across subjects for each of the three components of the study (computer task, buffet and fMRI). As part of the baseline visit, body weight and height were measured using a digital scale adjusted to the first decimal and a stadiometer, respectively. Subjects were also asked to complete the Three Factor Eating Questionnaire (TFEQ), which evaluates individual differences in eating behavior regarding dietary restraint, disinhibition and hunger [28]. All references in the text to eating behavior characteristics refer to scores in these three components of the TFEQ. The study protocol was approved by the Institutional Review Board (IRB) of Beth Israel Deaconess Medical Center. All subjects gave written informed consent before participation and received monetary compensation for their time spent in the study.

\section{RESULTS}

Stop-signal task (SST)

Table 1 shows summary statistics of overall task performance for the 30 participants. The version of the SST that we used was successful in achieving approximately 50\% inhibition accuracy, indicating that the staircase-tracking algorithm was adequate. Stop-signal reaction time (SSRT) values were within previously reported ranges [29].

Psychomotor performance. Mean Go reaction time (RT) was not influenced by condition, time or their interaction (all $p>0.3$ ). Overall, subjects did not differ in performance based on trial type (neutral/food) $(p=0.542)$, and no condition $x$ time $x$ trial type interaction was found $(p=0.923)$. When the effect of eating behavior characteristics was modeled, hunger significantly influenced the interaction condition $\mathrm{x}$ time $(p=0.025)$. Post hoc comparisons showed that subjects with higher hunger scores had more slowing of reaction times after intake of the MSG + broth [net change: upper tertile: $17.6 \pm 10.3$; lower tertile: $-10.4 \pm 11.1$ (ms)]. Controlling for the effect of eating behavior characteristics, the interaction condition $\mathrm{x}$ time $\mathrm{x}$ trial type reached borderline significance $(p=0.077)$. Specifically, intake of the MSG + broth tended to reverse the slowing of Go RT during food trials that was observed following the MSG - broth (Table 1), possibly attributed to a priming effect of the broth on attention toward food images.

Response inhibition. Figure 2a depicts overall results for SSRT, the main inhibition-related parameter of the task. In a general model, SSRT was not affected by time, condition, or trial type (all $p>0.25$ ). To account for general differences in motor speed that could have masked an effect, we performed an analysis of SSRT controlling for mean Go RT. This analysis revealed a significant condition $x$ time interaction $(p=0.033)$ : subjects had more reduction of SSRT following intake of the MSG+ broth [pre: 155.1, post: 154.4 (ms)], than with the MSG - broth [pre: 157.3, post: $162.4(\mathrm{~ms})]$. When eating behavior characteristics were considered, there was a condition $x$ time $x$ hunger interaction $(p=0.025)$ [net change: upper tertile: $1.5 \pm 9.2$; lower tertile: $-20.6 \pm 15.2$ (difference, ms)] that remained significant after adjusting for motor speed $(p=$ 0.042). The effect of MSG + broth on SSRT was also correlated with body mass index (BMI): subjects with higher BMI had more reduction of SSRT ( $r=-0.461, p=0.010)$ (Fig. 2b). This association was unrelated to Go RT (partial correlation adjusted for mean Go RT: $r=-0.457, p=0.013)$.

Inhibition accuracy (\% correct stops) was significantly affected by time only $(p=0.005)$, reflecting that subjects were better overall at inhibiting responses in the post-broth state. No interactions were observed. Eating behavior characteristics did not influence these results (all $p>0.2$ ).

Response time variability. There was a main effect of time on intra-individual coefficient of variation (ICV) $(p<0.001)$. Overall, subjects had less fluctuation of their performance in the postbroth state than in the pre-broth state. Modeling eating behavior characteristics revealed an interaction effect of condition $x$ time $x$ disinhibition $(p=0.025)$. Specifically, subjects with higher disinhibition scores had more reduction in response variability after intake of the MSG + broth (Fig. 2c), indicative of better ability to maintain a consistent level of attention over time [net effect: lower tertile: $0.08 \pm 0.07$; upper tertile: $-0.13 \pm 0.07$ (ratio difference)]. This effect was not trial type specific, and it was unrelated to 

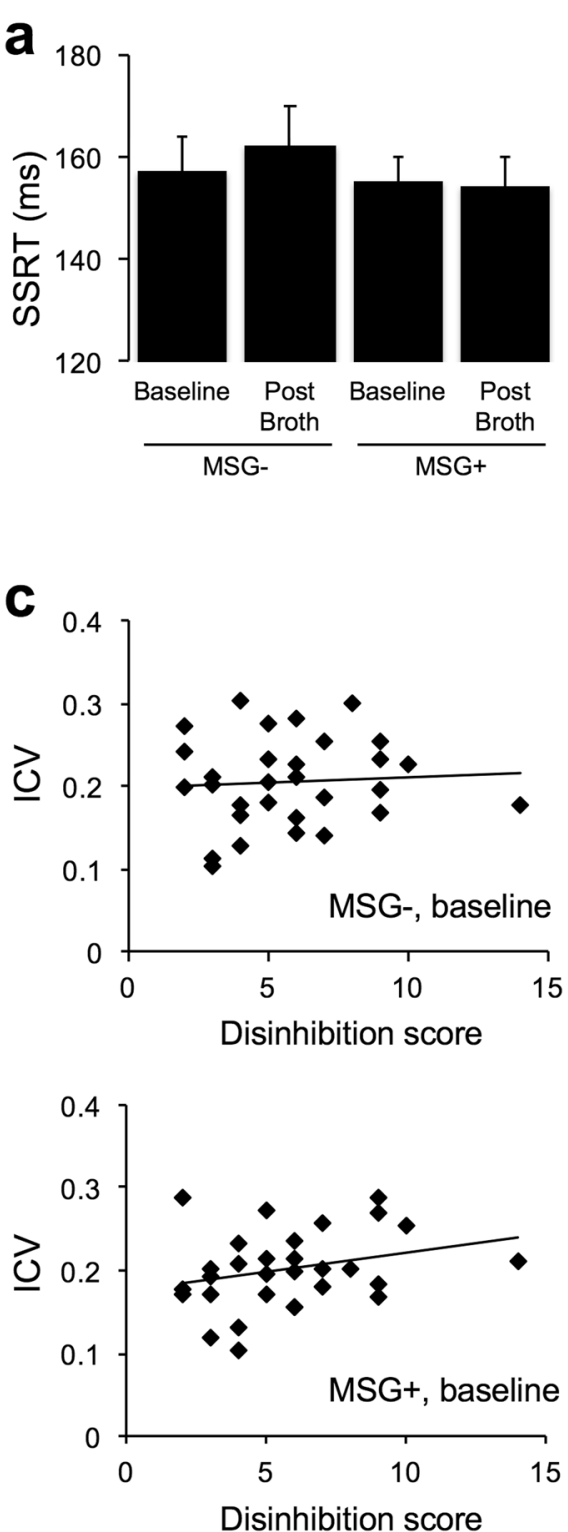

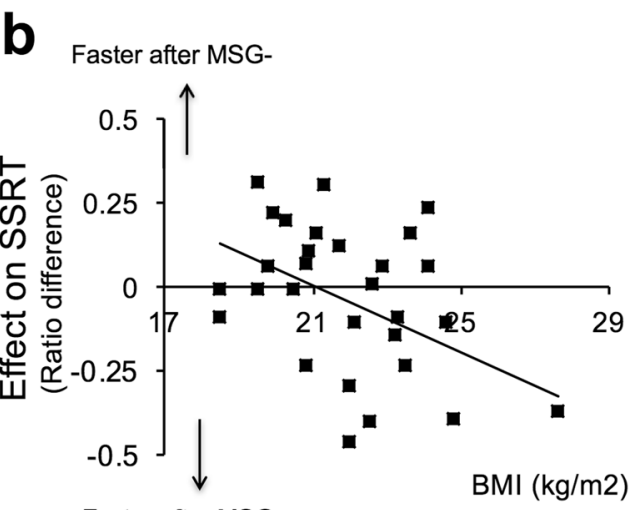

Faster after MSG+
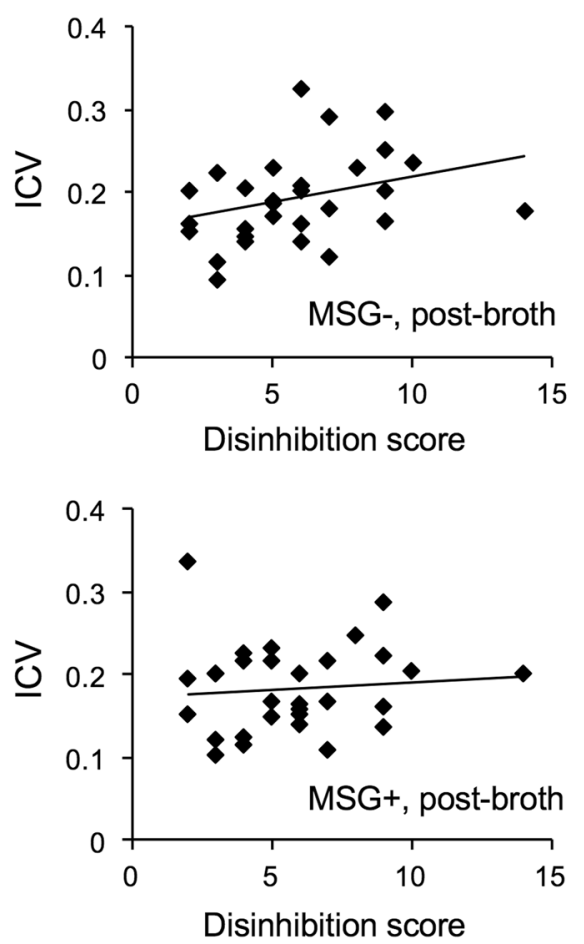

Fig. 2 a Stop signal reaction time (SSRT) changes across condition and time. Bars represent Mean \pm SEM. b Correlation between the effect of MSG + broth on SSRT and BMI $(p=0.010)$. Effect was estimated as the difference of post-broth/pre-broth ratios. c Interaction effect of disinhibition on intra-individual coefficient of variation (ICV) $(p=0.025)$

general differences in motor performance, as it remained significant after controlling for mean Go RT $(p=0.042)$.

\section{Buffet meal}

Total intake. Energy intake results are displayed in Fig. 3a. There were no differences in total consumption (MSG: $1086 \pm 130 \mathrm{kcal}$; MSG+: $1213 \pm 87 \mathrm{kcal}, p=0.243)$, i.e., within-subject change in intake after the MSG + broth overlapped zero (127 $\pm 104 \mathrm{kcal})$.

Macronutrients. Broth condition did not have an effect at the macronutrient level. Subjects consumed similar amounts of carbohydrates, protein, fiber, fat and saturated fat. This was observed both with the data expressed in total weight $(\mathrm{g})$ as well as in percentage of total calories (all $p>0.2$ ) (Fig. 3b).

Effect of individual variability. When we examined the role of eating behavior characteristics, we found an inverse correlation between disinhibition and change in saturated fat intake $(\mathrm{g})$ after MSG + broth consumption $(r=-0.616, p=0.015)$ : subjects with higher disinhibition scores consumed less saturated fat (Fig. 3c). This effect appeared to be selective, as we did not find any association with other macronutrients (all correlations $p>0.2$, aside from fat overall $r=-0.382, p=0.160$ ).

General aspects and food choice. General characteristics of the meal did not differ across conditions. This included meal duration, total number of events, and eating speed (all $p>0.4$ ). Broth condition did not influence consumption based on food categories, i.e., subjects ate similarly from all plates considering the effect of calorie density (low calorie/high calorie), taste (savory/sweet), or a combination of both. However, subjects with higher scores in disinhibition ate less from low-calorie savory food (appetizer and entrée plates combined) following the MSG + broth $(r=-0.570, p=0.027)$. No other effects were found with other categorizations of food choice, such as low calorie $(r=-0.427, p$ $=0.113)$, high calorie $(r=-0.298, p=0.280)$, or individual plate types. Only trend-level negative associations were observed for individual low calorie, savory plates (appetizer low: $r=-0.474, p$ 

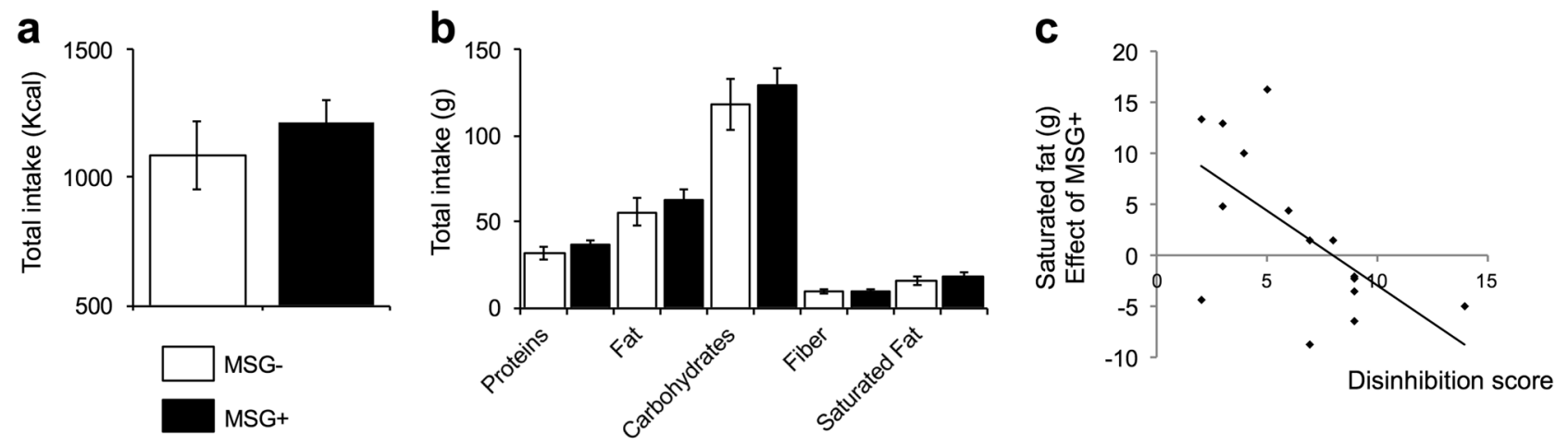

\section{d}
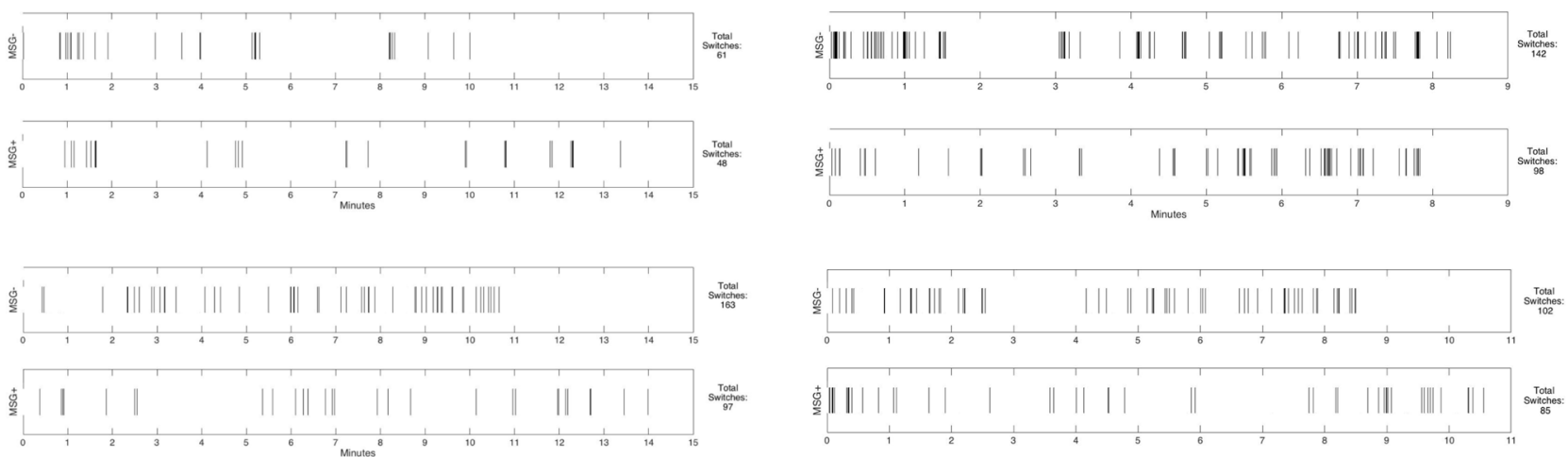

Fig. 3 a Ad libitum intake during the buffet meal test, total calories consumed. Bars represent mean \pm SEM. b Ad libitum intake during the buffet meal test, macronutrient data, expressed in total weight consumed (g). Bars represent mean \pm SEM. c Correlation between disinhibition score and the effect of MSG + on saturated fat intake during the buffet (total intake after MSG + broth minus total intake after MSG - ) ( $p=$ 0.015). d Fixation switching rate during the first third of the buffet meal in four representative subjects. Lines indicate each time there is a fixation switch. Upper rows represent MSG- condition and lower rows represent MSG+ condition

a

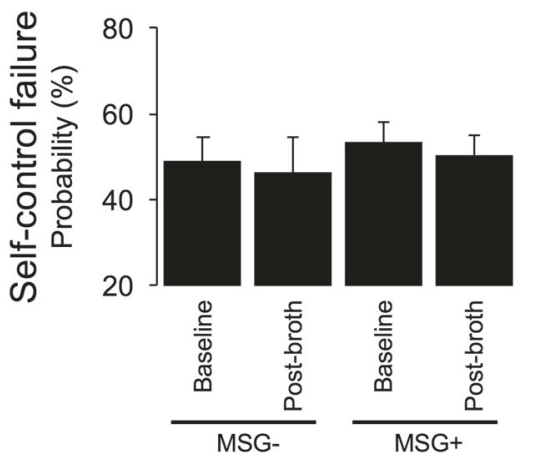

b

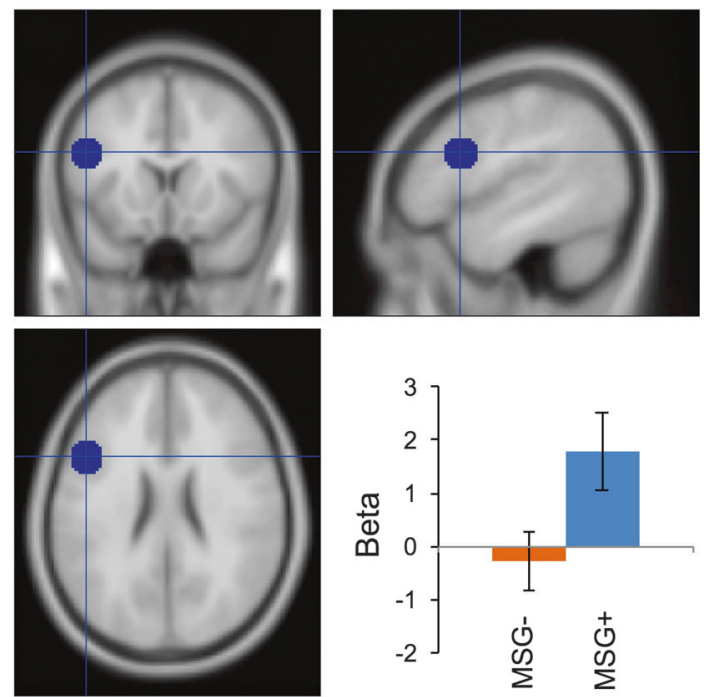

Fig. 4 a fMRI food choice task, behavioral findings. Data show the percentage of the time participants did not chose the healthy/unpalatable or chose the unhealthy/palatable food over the reference, neutral food (self-control failure). Bars represent Mean \pm SEM $\mathbf{b}$ Beta values in the left dorsolateral prefrontal cortex ROI showed greater activity following intake of the MSG + vs. following intake of the MSG- broth $(p=0.026)$

$=0.074$; entrée low: $r=-0466, p=0.080$; dessert low: $r=0.120, p$ $=0.671$; appetizer high: $r=-0.153, p=0.586$; entrée high: $r=$ $-0.144, p=0.609$; dessert high: $r=-0.372, p=0.172$ ).

Eye-tracking. We found a significant reduction in switching rate overall, i.e., when controlling for eating behavior characteristics ( $p$
$=0.006)$. Specifically, following intake of the MSG + broth, subjects had lower switching rates than after intake of the MSG- broth (MSG- $10.4 \pm 1.0$, MSG $+8.9 \pm 0.8$ ). This reduction effect occurred in 10 out of the 13 analyzed subjects and is illustrated in Fig. $3 \mathrm{~d}$. Other parameters did not show an effect, except for disinhibition, which was associated with the percentage of matches $(p=0.025)$. 
Specifically, subjects with higher disinhibition scores tended to have more fixations on the plates from which they were eating after the MSG - broth than after the MSG + broth.

fMRI

During the $\mathrm{fMRI}$ task (choice run), subjects did not differ in their performance between broth conditions. Specifically, the probability of failure during trials that required self-regulation remained at similar levels (MSG-: baseline 49.03\%, post-broth 46.2\%; MSG+: baseline 53.6\%, post-broth 50.3\%) $(p=0.954)$ (Fig. 4a). When we examined brain activation during those decision scenarios, we found that after the MSG+ broth, subjects had more activation in the left prefrontal cortex region of interest (ROI) previously linked to successful self-control during food choice (Wilcoxon Signed Ranks Test, $Z=-2.223, \quad p=0.026$ ) (Fig. 4b). In the whole-brain analysis, we also found more reduction after MSG+ in a range of areas that have been linked to increased motivation and attention to food, such as the cerebellum, precuneus and fusiform gyrus (Supplementary Table 1). Additional findings related to brain activations during health and tasting ratings and overall choice are reported in Supplementary Tables 2-4.

\section{Blinding success}

At the end of the study, subjects were asked to guess the order in which they thought they had received the broth conditions throughout the visits. $40.7 \%$ of participants were able to predict the order correctly. Thus, their guesses were not different from chance level $\left[x^{2}(1)=0.926, p=0.336\right]$, suggesting that blinding procedures were successful.

\section{DISCUSSION}

In the present study, we examined neurocognitive effects following intake of MSG that was ingested as part of a chicken broth preload. We evaluated participants with a computer-based inhibitory control task, a buffet meal with eye-tracking, and an fMRI food choice task. We found that intake of the MSG+ broth was associated with: (1) changes in key parameters of response inhibition in association with eating behavior characteristics, specifically improvements in subjects with propensity to overeat and gain weight, and (2) a decrease in saturated fat intake during the buffet meal in this same group of subjects. Additionally, we found (3) a general reduction in the rate of eye fixation switches between plates at the meal, and (4) increased activation, during dietary choice, of a left prefrontal cortex region related to successful self-control.

Our findings represent the first evidence to date regarding a potential effect of MSG on neurocognitive parameters that are relevant for the support of healthy eating behaviors and food choice. They also extend our understanding of how this umami substance may influence energy balance. However, due to the small sample size, these data should be interpreted as preliminary and future research should confirm our findings. Similar to previous research, and as hypothesized, we found that part of the effects of MSG only occurred or were stronger for women with high disinhibition or BMI. Specifically, our study replicated previous research that showed less intake of high-fat food following a preload with MSG, in association with disinhibition [12] or frank overweight/obesity [17]. However, we did not see a general impact on total kcal consumption as reported in two recent studies $[13,17]$. This disagreement may be related to subject characteristics, fasting hours, or the composition of the buffet, which in our case was constrained by design to two calorically conflicting menu sets (6 plates) vs. 16 options used in these prior studies. Regarding the influence of eating behavior characteristics, our study confirmed the already known link between disinhibition and a beneficial effect of MSG in the context of food intake and, for the first time, in the domain of neurocognition (inhibitory control). We should point out, however, that the effects that we found were not specific of trial type (food/ neutral images), indicating that they may have occurred through an influence on neurocognition in general, i.e. independent of food. As a novel contribution, we also found that hunger scores had an inverse association with improvements in response inhibition and general psychomotor performance after intake of MSG. The relevance of this finding is unclear, as the hunger component of the TFEQ has not been as extensively studied as disinhibition [30]. Hunger scores may reflect variability in internal signaling pathways more closely linked to homeostatic regulation of appetite, i.e., hypothalamic circuits [31], which are possibly more established and less prone to modulation via neurocognition. Additionally, recent data suggest that scores in the hunger factor of the TFEQ can be influenced by momentary hunger [32]. In our case, the TFEQ was administered at a baseline visit, which took place on a different day than the visits at which the outcomes were collected. For that baseline visit, subjects were not required to arrive under a specific window of fasting. Thus, it is also unclear how to interpret these findings under that perspective, due to inconsistent fasting states at the time of answering the questions. As another novel contribution, we evaluated subjects with complementary methodologies that captured changes in three domains: brain, cognition, and behavior. The direction of effects was consistent across modalities. Computer task and fMRI results were echoed by the eye-tracking data and, to some extent, food intake. In combination, our results suggest that the observed neurocognitive effects associated with MSG seem to transfer to real-life scenarios related to healthy eating behavior and food choice. However, more research is needed to confirm and replicate these findings.

Our results can be explained by priming of neural responses due to orosensory information, particularly signaling via glutamate receptors in the oral cavity, stomach, or a combination of both. Subjects were not able to distinguish the broths retrospectively, suggesting that taste, at least consciously, may have played only a minor role. However, we did not evaluate sensory aspects in detail, and the time delay between broth ingestion and outcome measures (15-30 min) makes both taste and post-ingestive effects plausible [33]. To our knowledge, no prior study has investigated the impact of taste or other orosensory characteristics of food on SST performance, and the effects of MSG signaling in the oral cavity on food intake are not well known, overall [17]. The SST changes that we observed are compatible with the hypothesized effect of MSG on neurocognition through the vagus nerve and ascending noradrenergic pathways (i.e. post-ingestive effects). Studies combining vagus nerve stimulation and the SST have shown SSRT improvements in patients who find clinical benefits from the device for epilepsy, which are believed to be mediated by activation of the locus coeruleus-noradrenergic system [25]. Additionally, based on accumulated data both in animals and humans, it is known that SST performance, specifically its SSRT and ICV measures, is prominently associated with the action of noradrenaline [34]. Our data also suggest that individuals with high predisposition to overeat and gain weight may be particularly responsive to the activation of this pathway with MSG, based on the profile of effects observed here, together with the past literature on SST and experimental research linking MSG to vagus nerve signaling in the stomach. It is unclear why subjects with this profile could benefit the most, but a growing body of research suggests that chronic intake of high-calorie diets, even without obesity, can lead to a disruption of vagus nerve sensitivity to peripheral signals, even though the detailed mechanisms remain unknown [35]. Also in support of a vagal mediation of the observed effects is the recently reported link between eating disinhibition and decreased vagal tone [36]. Assuming that individuals with high levels of disinhibition might already have 
some degree of dysfunction in the vagus nerve, it is possible that MSG, as used in our protocol, could have facilitated transmission in this pathway and thus benefited physiological regulatory mechanisms related to energy balance. Future studies should integrate measurements of the status of vagus nerve functioning such as heart rate variability to confirm this intriguing possibility.

There was an overall reduction in switching rate between plates during the buffet after intake of the MSG + broth. We believe this finding may be compatible with a better deployment of attentional control resources and/or less responsivity to the presence of palatable food cues, as it aligns well with both the general improvements in SST parameters and the effects in fMRI that we found. As the methodology that we used here was novel, it is difficult to extrapolate findings from past research. Using the same buffet meal test paradigm with no eye-tracking, we observed in the past that subjects who reported experiencing binge eating behaviors showed more bite switches between plates than healthy controls (unpublished data). However, successful self-regulation during food choice was associated with more gaze switching in a previous study that combined eyetracking with fMRI [37]. While our buffet scenario was designed to recreate the cognitive dilemma of choice between high- and lowcalorie food, subjects were eating freely in a relaxed setting and were not asked to make forced choices; thus it is unclear if we can extend these fMRI findings to the particular case of our study. The ability to maintain attention while exposed to salient food cues is a critical factor to guarantee goal-oriented behavior in general, and for the specific case of eating, to prevent food craving and overeating [38]. Neuroimaging studies have shown a link between attentional bias to food, underlying neural correlates of this bias, and changes in BMI over time [39], and a number interventions aimed at enhancing attentional control over food are under investigation to reduce overeating and body weight [e.g., [40]]. Another possible interpretation of our findings could be a general increase in the ability to focus attention during the buffet and avoid the distractibility driven by multiple choices. This possibility warrants further examination, as inattention during a meal is known to interfere with satiety mechanisms [41]. Future studies should clarify whether the identified effects of MSG on switching among plates represent any of these specific scenarios and should also address the overall long-term impact on food intake and satiety.

Last, the finding of higher activation in the left prefrontal cortex during the fMRI food choice task suggests that MSG intake induced a facilitation of neural substrates associated with successful self-control. However, this effect was seen without changes in task performance, i.e. choice performance remained at similar levels between conditions, indicating no behavioral improvement in self-control capacity. This suggests that the impact of MSG may not have been strong enough to translate into behavior during the task and only impacted its neural underpinnings. However, it is possible that the small sample size and limited number of self-control trials precluded identification of the behavioral effect. The brain changes that we observed here are compatible with a facilitation of executive processes, based on previous research [27] and on the combination of findings in the other components of this study. A seminal fMRI brain investigation that studied the representation of the taste of umami substances, including MSG, found more activation in the dorsal sector of the anterior cingulate cortex (dACC), compared with activation representative of glucose taste [21]. While our study cannot be directly linked with this finding, the $\mathrm{AACC}$ is highly connected with the lateral sector of the prefrontal cortex, particularly during cognitive-executive performance, and we found more activation in a region overlapping the dACC when subjects were giving higher scores for the health characteristics of food, in the prebroth (baseline) state. Additionally, in line with the overall hypothesis of our study, markers of vagus nerve activation, such as heart rate variability, have been linked with self-regulatory capacity and its neural correlates, suggesting a convergence of these autonomic and cognitive pathways [42].

An additional limitation of our study pertains to the use of MSG only in the broth. While other umami substances, such as inosinate (IMP) and guanylate (GMP), have synergistic effects with glutamate to enhance the intensity of umami taste, many studies have used MSG alone as a proxy of umami taste. Specifically, our decision to use a broth with MSG only, i.e., without additional umami substances, was based on previous studies that used chicken broth [13, 19], vegetable soup [17], and milk-based preloads [12]. These studies identified effects of MSG, added in isolation, on appetite and food intake, using concentrations that were similar to the one we used here. As the purpose of our study was to examine potential neurocognitive mechanisms underlying these effects, we chose to replicate preload (broth) composition as closely as possible. Future studies should examine whether our results can be extrapolated to the case of other umami substances or combinations. Our study has also a number of more general implications related to the connection between dietary intake and neurocognition. Previous research has shown that the vagus nerve-brainstem-noradrenergic system pathway is also involved in the effects that are seen with a diet rich in protein [43]. Whether the impact on neurocognition that we observed here can be translated to more general scenarios of protein intake and specific diet compositions remains unknown. Also, the tradition of eating a soup as a starter at the beginning of a meal in many cultures around the world may be associated with beneficial effects in the regulation of intake, through the involvement of neurocognitive effects, based on the results of our study. While our study is not free of limitations, it represents the first attempt to connect neurocognition and appetite in this area of research, and we hope that it will spur future investigations integrating these traditionally separated fields of research.

In sum, our study suggests that MSG intake as part of a preload is associated with an enhancement of cognitive executive processes, specifically in the domain of inhibitory control and in women with vulnerability to obesity. While our findings should be considered preliminary due to the limited sample size and marginal levels of significance, they point to potential facilitating effects of glutamate (MSG) on cognitive executive processes that are relevant for the support of healthy eating behaviors and food choice.

\section{ACKNOWLEDGEMENTS}

We thank kitchen and nurse support personnel from the Clinical Research Center of Beth Israel Deaconess Medical Center and technical assistant and advice from Dr. Ron Killiany and Andrew Ellison from the Center for Biomedical Imaging, Boston University Medical Center. We thank all participants who took part in this study for their time and help. This study was supported by a grant from Ajinomoto Inc., which had no role in the design, analysis or writing of this article, and the Harvard Catalyst/ Harvard Clinical and Translational Science Center (8UL1TR000170-05 and 1UL1 TR001102-01).

\section{ADDITIONAL INFORMATION}

The online version of this article (https://doi.org/10.1038/s41386-018-0044-6) contains supplementary material, which is available to authorized users.

Competing interests: The authors declare no competing interests.

Publisher's note: Springer Nature remains neutral with regard to jurisdictional claims in published maps and institutional affiliations.

\section{REFERENCES}

1. Ikeda K. New seasonings. Chem Senses. 2002;27:847-9.

2. Lindemann B, Ogiwara Y, Ninomiya Y. The discovery of umami. Chem Senses. 2002;27:843-4.

3. Ackroff K, Sclafani A. Flavor preferences conditioned by dietary glutamate. Adv Nutr. 2016;7:845S-852S. 
4. Kondoh T, Mallick HN, Torii K. Activation of the gut-brain axis by dietary glutamate and physiologic significance in energy homeostasis. Am J Clin Nutr. 2009;90:832S-837S.

5. Torii $\mathrm{K}$, Uneyama $\mathrm{H}$, Nakamura E. Physiological roles of dietary glutamate signaling via gut-brain axis due to efficient digestion and absorption. J Gastroenterol. 2013:48:442-51.

6. Kurihara K. Umami the fifth basic taste: history of studies on receptor mechanisms and role as a food flavor. BioMed Res Int. 2015;2015:189402.

7. Tsurugizawa $T$, Uematsu A, Nakamura E, Hasumura M, Hirota $M$, Kondoh $T$, et al. Mechanisms of neural response to gastrointestinal nutritive stimuli: the gut-brain axis. Gastroenterology. 2009;137:262-73.

8. Tsurugizawa $\mathrm{T}$, Uneyama $\mathrm{H}$, Torii $\mathrm{K}$. Brain amino acid sensing. Diabetes Obes Metab. 2014;16:41-48.

9. Uneyama H, Niijima A, San Gabriel A, Torii K. Luminal amino acid sensing in the rat gastric mucosa. Am J Physiol Gastrointest Liver Physiol. 2006;291:G1163-1170.

10. Tsurugizawa T, Kondoh T, Torii K. Forebrain activation induced by postoral nutritive substances in rats. Neuroreport. 2008;19:1111-5.

11. Kondoh T, Torii K. MSG intake suppresses weight gain, fat deposition, and plasma leptin levels in male Sprague-Dawley rats. Physiol Behav. 2008;95:135-44.

12. Finlayson G, Bordes I, Griffioen-Roose S, de Graaf C, Blundell JE. Susceptibility to overeating affects the impact of savory or sweet drinks on satiation, reward, and food intake in nonobese women. J Nutr. 2012;142:125-30.

13. Imada T, Hao SS, Torii K, Kimura E. Supplementing chicken broth with monosodium glutamate reduces energy intake from high fat and sweet snacks in middle-aged healthy women. Appetite. 2014;79:158-65.

14. Masic U, Yeomans MR. Does monosodium glutamate interact with macronutrient composition to influence subsequent appetite? Physiol Behav. 2013; 116-7:23-29.

15. Masic U, Yeomans MR. Monosodium glutamate delivered in a protein-rich soup improves subsequent energy compensation. J Nutr Sci. 2014a;3:e15.

16. Masic U, Yeomans MR. Umami flavor enhances appetite but also increases satiety. Am J Clin Nutr. 2014b;100:532-8.

17. Miyaki T, Imada T, Hao SS, Kimura E. Monosodium L-glutamate in soup reduces subsequent energy intake from high-fat savoury food in overweight and obese women. Br J Nutr. 2016;115:176-84.

18. Rogers PJ, Blundell JE. Umami and appetite: effects of monosodium glutamate on hunger and food intake in human subjects. Physiol Behav. 1990;48:801-4.

19. Carter BE, Monsivais P, Perrigue MM, Drewnowski A. Supplementing chicken broth with monosodium glutamate reduces hunger and desire to snack but does not affect energy intake in women. Br J Nutr. 2011;106:1441-8.

20. Bellisle F. Experimental studies of food choices and palatability responses in European subjects exposed to the Umami taste. Asia Pac J Clin Nutr. 2008;17:376-9.

21. de Araujo IE, Kringelbach ML, Rolls ET, Hobden P. Representation of umami taste in the human brain. J Neurophysiol. 2003;90:313-9.

22. Bush G, Luu P, Posner MI. Cognitive and emotional influences in anterior cingulate cortex. Trends Cogn Sci. 2000;4:215-22.

23. Berthoud HR, Neuhuber WL. Functional and chemical anatomy of the afferent vagal system. Auton Neurosci. 2000;85:1-17.
24. Martin CO, Denburg NL, Tranel D, Granner MA, Bechara A. The effects of vagus nerve stimulation on decision-making. Cortex. 2004;40:605-12.

25. Schevernels $H$, van Bochove ME, De Taeye L, Bombeke K, Vonck K, Van Roost D, et al. The effect of vagus nerve stimulation on response inhibition. Epilepsy Behav. 2016;64:171-9.

26. Cao B, Wang J, Shahed M, Jelfs B, Chan $\mathrm{RH}$, Li Y. Vagus nerve stimulation alters phase synchrony of the anterior cingulate cortex and facilitates decision making in rats. Sci Rep. 2016;6:35135.

27. Hare TA, Camerer CF, Rangel A. Self-control in decision-making involves modulation of the vmPFC valuation system. Science. 2009;324:646-8.

28. Stunkard AJ, Messick S. The three-factor eating questionnaire to measure dietary restraint, disinhibition and hunger. J Psychosom Res. 1985;29:71-83.

29. Congdon E, Mumford JA, Cohen JR, Galvan A, Canli T, Poldrack RA. Measurement and reliability of response inhibition. Front Psychol. 2012;3:37.

30. Bryant EJ, King NA, Blundell JE. Disinhibition: its effects on appetite and weight regulation. Obes Rev. 2008;9:409-19.

31. Yao L, Li W, Dai Z, Dong C. Eating behavior associated with gray matter volume alternations: a voxel based morphometry study. Appetite. 2016;96:572-9.

32. Yeomans MR, McCrickerd K. Acute hunger modifies responses on the three factor eating questionnaire hunger and disinhibition, but not restraint, scales. Appetite. 2017;110:1-5.

33. Livingstone $B E$, Robson PJ, Welch RW, Burns AA, Burrows MS, McCormack C. Methodological issues in the assessment of satiety. Scand J Nutr. 2000;44:98-103.

34. Eagle DM, Bari A, Robbins TW. The neuropsychopharmacology of action inhibition: cross-species translation of the stop-signal and go/no-go tasks. Psychopharmacology. 2008;199:439-56.

35. de Lartigue $\mathrm{G}$. Role of the vagus nerve in the development and treatment of dietinduced obesity. J Physiol. 2016;594:5791-815.

36. Young HA, Watkins $H$. Eating disinhibition and vagal tone moderate the postprandial response to glycemic load: a randomised controlled trial. Sci Rep. 2016;6:35740.

37. van der Laan LN, de Ridder DT, Charbonnier L, Viergever MA, Smeets PA. Sweet lies: neural, visual, and behavioral measures reveal a lack of self-control conflict during food choice in weight-concerned women. Front Behav Neurosci. 2014;8:184.

38. Higgs S. Cognitive processing of food rewards. Appetite. 2016;104:10-17.

39. Yokum $\mathrm{S}, \mathrm{Ng} J$, Stice E. Attentional bias to food images associated with elevated weight and future weight gain: an fMRI study. Obesity. 2011;19:1775-83.

40. Bazzaz MM, Fadardi JS, Parkinson J. Efficacy of the attention control training program on reducing attentional bias in obese and overweight dieters. Appetite. 2017; 108:1-11.

41. Robinson E, Aveyard P, Daley A, Jolly K, Lewis A, Lycett D, et al. Eating attentively: a systematic review and meta-analysis of the effect of food intake memory and awareness on eating. Am J Clin Nutr. 2013;97:728-42.

42. Maier SU, Hare TA. Higher heart-rate variability is associated with ventromedial prefrontal cortex activity and increased resistance to temptation in dietary selfcontrol challenges. J Neurosci. 2017;37:446-55.

43. Tome D, Schwarz J, Darcel N, Fromentin G. Protein, amino acids, vagus nerve signaling, and the brain. Am J Clin Nutr. 2009;90:838S-43S. 